\title{
Participación de la mujer en el cooperativismo costarricense (1989-2018)
}

\author{
Roxana Sánchez Boza \\ Catedrática de la Universidad de Costa Rica
}

Sumario: Siglas. Introducción. 1. Contexto de la equidad e igualdad de género en el cooperativismo costarricense. Bases legales y de derecho convencional. La política nacional para la igualdad y la equidad de género (PIEG) de Costa Rica. 2. Los principios cooperativos de gestión democrática, participación económica y educación, capacitación e información como fundamento de los derechos de las mujeres: dentro de las cooperativas y en los entes de representación y defensa del cooperativismo. 3. La visibilización de la participación de las mujeres dentro de los entes cooperativos en el período 1989-2012. 3.1. Limitada presencia en los cargos de dirección. 3.2. Los hombres si tienen capacidades innatas para dirigir a las mujeres. 3.3. Ubicación de la mayoría de las mujeres en la educación cooperativa. 4. Relación con los puestos ocupados por varones en instituciones públicas. Incidencia de las políticas públicas del país en relación con las oportunidades económicas y políticas ofrecidas a las mujeres. 5. Reforma a la ley de asociaciones cooperativas para introducir la equidad e igualdad de género. Conclusiones. Bibliografía.

Resumen: La historia de la participación de la mujer en el Cooperativismo costarricense se puede escribir a partir de finales de la década de los ochenta, época en la que la mujer y su aporte al cooperativismo permaneció invisibilizado. Durante tres décadas un reducido grupo de mujeres, con una visión clara sobre la naturaleza jurídico-social de las cooperativas, así como de sus principios, han dado una lucha por lograr una participación político-económica en igualdad de condiciones y con equidad de género, dentro de la organización cooperativa. Los resultados aún no han sido positivos, pese a las políticas públicas del Estado costarricense en esa ruta y un contexto general favorable del cooperativismo mundial, dado que la Alianza Cooperativa Internacional que promueve el establecimiento de una igualdad y equidad a favor de las mujeres cooperativistas en los países de América.

Palabras clave: mujeres cooperativistas - participación político-económica- igualdad y equidad en los entes cooperativos. 
Abstract: The history of the participation of women in Costa Rican cooperativism can be written from the late 1980s, when woman and her contribution to cooperativism remained invisible. During three decades, a small group of women, with a clear vision of the legal-social nature of cooperatives, as well as their principles, have given the struggle to achieve an equitable political-economic participation within the cooperative organization. The results have not yet been positive, despite the public policies of the Costa Rican State in this route and a general favorable context within the global cooperativism movement, where the International Cooperative Alliance promotes the establishment of equality and equity for the cooperative women in the countries of America.

Keywords: Women cooperative members- Political-economic participation-equality and equity in cooperative entities. 


\section{Siglas}

Amucode $\quad$ Asociación de Mujeres en Cooperación para el Desarrollo.

BPDC Ley del Banco Popular y Desarrollo Comunal.

Cenecoop R.L. Centro Nacional de Estudios Cooperativos R.L.

CNC Consejo Nacional de Concesiones.

Conamujer Comité Nacional de las Mujeres Cooperativas.

CONACOOP Consejo Nacional de Cooperativas. INFOCOOP Instituto Nacional de Fomento Cooperativo.

LAC

PIMA

SeNARA Ley de Asociaciones Cooperativas. Programa Integral de Mercadeo Agropecuario. Servicio Nacional de Aguas de Riego.

\section{Introducción}

El Cooperativismo en Costa Rica ya cumplió el siglo de existencia y con apoyo del Estado costarricense, sobre todo en las décadas de los años setenta y ochenta, se dio un salto cualitativo con la creación tanto del Instituto Nacional de Fomento Cooperativo, INFOCOOP como ente estatal con funciones de financiamiento, capacitación y fiscalización de las cooperativas de primer y segundo grado como del Consejo Nacional de Cooperativas CONACOOP definido legalmente como ente público no estatal de representación y defensa del cooperativismo.

La mayoría de las cooperativas creadas fueron para desarrollar el sector agroindustrial, de ahorro y crédito y de vivienda, sectores reproductores del modelo patriarcal donde el hombre es el principal obligado a atender las necesidades de la familia, y por lo tanto las mujeres, no tenían acceso a los medios de producción ni podían contraer deudas como deudoras por créditos personales o por la adquisición de una vivienda. El panorama cambió con la ampliación de la base cooperativa por el surgimiento de múltiples cooperativas de ahorro y crédito en el sector educativo, formado en su mayoría por maestras y profesoras, género en el cuál recaía y recae, en su mayor parte, la labor docente del país.

El aumento de la participación de la mujer dentro del cooperativismo costarricense fue notorio a inicios de los años noventa. De tal modo, que algunos grupos organizados de mujeres cooperativistas dieron inicio a un movimiento caracterizado por buscar una mayor participación en los entes de representación y defensa del Cooperativismo así como en los órganos sociales de las cooperativas de primer y segundo grado, conocidas como uniones, federaciones y organismos auxiliares del cooperativismo. 
Los datos que se han podido recuperar de diferentes instrumentos estadísticos permiten conocer parcialmente la posición política de la mujer dentro de la organización cooperativa. Por otro lado, también algunas autoras han dado sus opiniones en la búsqueda de ofrecer datos de tipo cualitativo, pero no ha existido incidencia de esos estudios, pues las mujeres se mantienen en desventaja porque por no han logrado llegar a puestos de la cúpula del Cooperativismo y tampoco hay conciencia dentro del mismo grupo femenino de abrir brechas para sus seguidoras.

\section{Contexto de la equidad e igualdad de género en el cooperativismo costarricense. Bases legales y de derecho convencional. La política nacional para la igualdad y la equidad de género (PIEG) de Costa Rica}

En Costa Rica existen múltiples leyes que buscan la protección jurídica y económica de las mujeres, una de estas corresponde a la ley que incorporó la Convención para la Eliminación de toda Forma de Discriminación contra las Mujeres (CEDAW, aprobada por la Asamblea Legislativa, en 1984, y la ley más significativa para el desarrollo de instrumentos legales y el establecimiento de mecanismos de ejecución de políticas, modificación de la normativa legal atinente y desarrollo de planes dirigidos a conseguir los objetivos de esa convención así como normas convencionales afines.

Pese una extensa doctrina y jurisprudencia de la Sala Constitucional que interpreta extensivamente todo lo relativo a la protección de los Derechos Humanos, según artículos 7 y 48 de la Constitución Política, las mujeres en el Cooperativismo costarricense siguen luchando por obtener un trato más equitativo en cuanto a la distribución de puestos y beneficios dentro de las cooperativas así como en las instituciones públicas donde el Cooperativismo tiene representación.

El entorno legislativo del país ha sido favorable al reconocimiento de los derechos políticos y económicos de la mujer: Ley 8322 promulgada en el año 2002, que reforma la Ley del Banco Popular y DesarroIlo Comunal, que estableció la paridad en todas las estructuras de decisión del mencionado Banco; Ley 8765 de 2009 correspondiente al Código Electoral que estableció el cumplimiento de la paridad en todas las estructuras de decisión de los partidos políticos y en todas las nóminas de elección popular; Ley n. 8901 que reforma varias leyes específicas obligando al cumplimiento de la paridad en las Juntas Directivas de diferentes sectores sociales (Asociaciones Civiles, Asociaciones Solidaristas, Asociaciones Comunales y Sindicatos). 
En el contexto centroamericano y sudamericano tanto la Confederación de Cooperativas de Centroamérica, del Caribe y Sudamérica así como la sede de la Alianza Cooperativa Internacional para América, han desarrollado diversas iniciativas y programas en aras de lograr la equidad e igualdad de género. En el caso de la primera existe el Comité Regional de la Mujer Cooperativista que actúa a través del Programa Regional de Equidad Género y Generacional (PREG), cuyo objetivo general es fomentar estrategias y acciones de participación igualitaria de género y generacional en el quehacer cooperativo. Y como indica en la página web de la Confederación, lo hace por medio del fortalecimiento de la presencia y acción de la dirigencia regional de las mujeres cooperativistas, por medio de diversas acciones como es asegurar la capacidad estratégica y el análisis compartido del CRMC; con el fomento de una participación visible de la dirigencia de las mujeres en los cónclaves cooperativistas de la región y abriendo espacios para el cumplimiento de políticas de igualdad en las cooperativas y respaldando el desarrollo de una certificación de igualdad de género (CCA-CA, s.a. página web).

En un nivel macro para América, se encuentra la plataforma de acción de mujeres cooperativistas del continente americano, como base del trabajo del Comité regional americano de mujeres cooperativistas (CRAMC-ACI AMERICAS).

Si bien Costa Rica es signataria de múltiples convenios de protección de los Derechos Humanos de la Mujer y a ha promulgado diversas leyes que los reconoce, es hasta el siglo XXI, que se elaboró una Política Nacional para la lgualdad y la Equidad de Género (PIEG), desde el 2007, como compromiso del Estado de avanzar en la igualdad de oportunidades y derechos entre mujeres y hombres, en la década de 2007-2017. Tal política se dio como una propuesta de cambio social de las fuentes de desigualdad, que obstaculizan el disfrute de oportunidades y derechos a la mitad de la población del país. La institución pública a cargo de su elaboración y ejecución es el Instituto Nacional de la Mujer INAMU (2007, p. 6). La PIEG se sustentó en tres grandes núcleos de acción estratégica: autonomía económica, participación política y cambio cultural. De importancia es la definición del concepto de igualdad y del concepto de equidad de género para efectos de la ejecución de la Política. Así en el enfoque de igualdad de género se parte del concepto de género como base de la comprensión y análisis de los significados, las relaciones y las identidades construidas socialmente producto de las diferencias biológicas entre los sexos. El enfoque de igualdad parte de la ausencia del reconocimiento de los derechos de las mujeres (p. 13). 
La igualdad social no se expresa en usos y costumbres socialmente vigentes, por el contrario, opera un doble discurso que reconoce derechos por un lado y los niega por otro, cuando se trata de concretar acciones que implican dar a las mujeres la posibilidad de ejercerlos a plenitud. (2007, p. 24)

Dentro de los objetivos de la Política se encuentra el fortalecimiento de la participación política de las mujeres y al logro de una democracia paritaria que toma como punto de partida para su planteamiento, los avances realizados al respecto, en el país, pero resalta la persistencia de subrrepresentación política de las mujeres en todos los espacios de toma de decisiones públicos y privados, debido en parte a que la legislación electoral a favor de los derechos políticos de las mujeres no siempre ha sido acatada y ha requerido del desarrollo de jurisprudencia adicional por parte del Tribunal Supremo de Elecciones (p. 75). La Política plantea la promoción paritaria en posiciones de toma de decisión y representación política, mediante la promoción de cambios normativos y desarrollo de jurisprudencia y procedimientos que aseguren la correcta aplicación de las leyes vigentes relativas a la participación política de las mujeres.

\section{Los principios cooperativos de gestión democrática, participación económica y educación, capacitación e información como fundamento de los derechos de las mujeres: dentro de las cooperativas y en los entes de representación y defensa del cooperativismo}

De los siete principios cooperativos revisados, modificados y aprobados por la Asamblea de la Alianza Cooperativa Internacional, en Manchester en 1995, interesa destacar por su relevancia para este estudio, el segundo, el tercer y el quinto, dejando claro que no es posible hacer su interpretación desprendiéndolos del cuerpo de Valores y Principios cooperativos que forman las bases de la filosofía cooperativista, pero que se tomó esa decisión por razones de espacio en el desarrollo de esta contribución.

Dentro de los instrumentos aprobados por la $\mathrm{ACl}$ para lograr un mejor marco legal para el Cooperativismo Latinoamericano se encuentra la Ley marco para cooperativas de América $(2009$, p. 4), donde se acoge la definición de cooperativa y los principios cooperativos, reconocidos y contenidos en la Declaración sobre la Identidad, así como en la Recomendación N. ${ }^{\circ} 193$ de la Organización Internacional del Tra- 
bajo, tomando asimismo en cuenta los documentos de otros organismos internacionales que en conjunto forman del Derecho Cooperativo Internacional, y dentro del cual no se hace especial referencia a la participación en condiciones de igualdad y equidad de las mujeres cooperativistas.

En relación con el segundo Control democrático de los miembros, en $1995 \mathrm{ACl}$ introduce la aplicación de este principio tanto para «hombres y mujeres», sin embargo, entre los comentarios se ve la dificultad de los comentaristas al texto del principio para incorporar a las mujeres (Eguía, F. y MacPherson, I., 2009, pp. 49, 158-167). Igual omisión se encuentra en el desarrollo del Tercer principio cooperativo relacionado con la «Participación económica de los socios», desarrollado por los autores mencionados (2009, pp. 50-53 y 167-174) y el maravilloso Quinto principio «Educación, capacitación e información» (2009, pp. 54-56, 181-188) que se refiere a «los socios, los jóvenes, los dirigentes». Si bien en el 2012 se dictan las Notas interpretación de los Principios Cooperativos que retoman a los dos segmentos humanos del Cooperativismo, cuando se refieren a la responsabilidad de hombres y mujeres cuando se expresa «Los hombres y las mujeres que ejercen como representantes elegidos son responsables respecto a todos los miembros». Se considera de importancia que los miembros electos que ocupen puestos de responsabilidad dentro de una cooperativa sean un buen reflejo de la diversidad de sus afiliados y si indica

(...) que si no lograre, será necesario adoptar medidas positivas para animar a los integrantes de las secciones infrarrepresentadas a que se presenten a las elecciones. Si existen barreras para ciertos grupos de miembros - como por ejemplo, mujeres - a la hora de presentarse a elecciones, habrá que introducir los cambios que corresponda para superar esa exclusión de las secciones de miembros en desventaja que les impide optar a cargos electos. (p. 18)

El interés por establecer situaciones de ventaja a las mujeres como grupo infra representado, no se mantiene presente en cuanto el ofrecimiento de oportunidades de educación y formación a los miembros nuevos como si se piensa en cuanto a los jóvenes. Y la referencia a la equidad se introduce cuando se considera que en el tercer principio, está equiparado a la justicia en el trato equitativo de todos los miembros (2012, p. 30). En el Cooperativismo destaca el segundo principio cooperativo que contiene aspectos de participación de las mujeres dentro de las organizaciones cooperativas; el tercer principio sobre gestión democrática y el quinto sobre educación y formación cooperativa. 
El Cooperativismo americano aprobó en 1997, la Plataforma de acción de mujeres cooperativistas del Continente Americano, en cuyos enunciados han reforzado la aspiración de la $\mathrm{ACl}$ de contribuir al cambio en las relaciones desiguales e injustas entre los géneros al interno de las cooperativas. Promover la creación de condiciones que garanticen una participación efectiva en las cooperativas así como en la toma de decisiones en todos los niveles y esferas. Diseñar mecanismos y estrategias equitativas de representación y acceso a la toma de decisiones y a ocupaciones productivas y dignamente remuneradas. Hacer visibles los aportes de las mujeres al desarrollo de las cooperativas, fortaleciendo los medios que permitan a las mujeres acceso al financiamiento y a los espacios de participación en la definición de las políticas económicas. Un nivel legal para facilitar recursos para investigación y análisis de las leyes que limitan, discriminan o impiden la participación de la mujer, así como para la formulación de proyectos, anteproyectos en el nivel institucional o gubernamental en cada país que contribuyan a la equidad (s.a. p. 10).

En el Cooperativismo costarricense se han aplicado ha aplicado diferentes censos para conocer, por medio de técnicas cuantitativas y cualitativas las modalidades de participación de los cooperativistas en los diferentes entes cooperativos. Cuatro diagnósticos tomaron en cuenta a toda la población cooperativa, ya sea a través de muestras o considerando todo su universo, y un Diagnóstico exclusivo para conocer y medir la participación de la mujeres en tales entes.

Antes de la revisión y modificación de algunos de los contenidos de los Principios Cooperativos, por parte de la ACl, en 1995, en Costa Rica, ya se había realizado el $V$ Congreso Cooperativo, con fecha de setiembre de 1987, foro en el cual acordó dar un mandato al Consejo Nacional de Cooperativas (CONACOOP) de crear una Comisión de mujeres, para permitir una mayor participación de la mujer dentro del Cooperativismo. La tarea de mayor rango establecida para el CONACOOP fue la realización de un diagnóstico en busca de determinar, con datos reales y actuales, el grado de participación de las mujeres en el Cooperativismo.

Con el Diagnóstico de 1988, se producen los primeros datos de orden científico de la historia del cooperativismo costarricense, impulsado por el Consejo Nacional de Cooperativas. En enero de 1988 se formó la Comisión mencionada y un año después se elaboró y aplicó el diagnóstico, con el apoyo de la Universidad de Costa Rica. La integración de la Comisión fue con mujeres cooperativistas que formaban parte del Plenario del CONACOOP y se sumaron representantes de la Unión nacional de cooperativas R.L., del Centro de estudios y capacitación cooperativa R.L., el Instituto nacional de fomento cooperativo y la Comisión permanente 
de cooperativas de autogestión. Durante 1989 la Alianza Cooperativa Internacional otorgó algunos recursos económicos a la Comisión y se varió su nombre a Comité nacional de la mujer cooperativista.

Los objetivos del Comité se han mantenido desde su creación y en resumen están dirigidos a lograr la plena incorporación y participación activa de las mujeres en el movimiento Cooperativo, a la par del reconocimiento de su dignidad como persona humana; existe un objetivo para capacitarlas en transferencia de tecnología, acorde con la actividad de su cooperativa y procurar la obtención de recursos para la buena marcha y ejecución de los programa de las mujeres cooperativistas.

En la 1991, la Asociación Programa Nacional de Asesoría y Capacitación para la Mujer, APROMUJER, organización de mujeres externa a la estructura política del Cooperativismo, también contribuyó con otro diagnóstico que complementó el referido de 1987. Ese diagnóstico no se hizo comparación con la cantidad de hombres distribuidos en los órganos sociales de las cooperativas pero da porcentajes de la participación de las mujeres que evidencian su baja participación política. Según indica APROMUJER (1991, p. 13) no hubo mucha colaboración con la aplicación de las encuestas, y se reflejó el poco interés existente en el Cooperativismo de los 90, en relación con el tema de género y participación de la mujer en sus estructuras político-sociales. El diagnóstico se basó en la formulación de cinco preguntas en torno a la participación de las mujeres dentro de los órganos sociales y en la gerencia de las cooperativas.

\section{Cuadro uno}

\section{Participación en órganos directivos}

\begin{tabular}{lcc}
\hline \multicolumn{1}{c}{ Órganos sociales } & Hombres & Mujeres \\
\hline Consejo de Administración & 826 & 127 \\
Comité de Vigilancia & 382 & 60 \\
Comité Educación y Bienestar Social & 276 & 172 \\
Comité de Crédito & 262 & 61 \\
\hline
\end{tabular}

Fuente: Elaboración propia con datos tomados de Cuadro 12 de Diagnóstico de APROMUJER 1991, p. 24.

El diagnóstico ofreció varios datos relacionados con la participación de hombres y mujeres en diferentes órganos cooperativos del Consejo Nacional de Cooperativas. Así en el cuadro uno se refleja una participación del Plenario de mujeres, en el período 1991-1993, que se duplicó, 
al pasar de 3 a 6 propietarias elegidas. Mientras que en las suplencias en el sector industrial no se eligió a ninguna mujer (1991, p. 9). En el Directorio del CONACOOP, en el período de 1979 a 1981 de siete miembros no se nombró a ninguna mujer. En los siguientes períodos de 1981 hasta 1993, de los miembros nombrados por períodos de 2 años, sólo una mujer se mantuvo como participante en ese cuerpo directivo (APROMUJER 1991, p. 15).

En cuanto a los asociados y asociadas, en 1989 de 289 cooperativas encuestadas, se reflejó una participación de 156.430 hombres y de 86.659 mujeres. Mientras en 1991, se encuestaron 129 cooperativas, y la participación de mujeres fue de 50.412 y de hombres 91.462 (APROMUJER, p. 23). Al final se dan conclusiones generales de los efectos de las características de la sociedad costarricense en el Cooperativismo, de tal modo que las participantes que dirigieron la aplicación y análisis del diagnóstico, consideraron que las actitudes discriminatorias cristalizadas por la vida diaria, tienen una resistencia al cambio, y una elasticidad muy fuerte, que reflejaron el decrecimiento en el número de mujeres gerentes dentro del sector, porque, afirmaron, que si bien no se negaba abiertamente el trabajo, también era cierto que no se creaban las facilidades para realizarlo (APROMUJER, p. 25).

\section{La visibilización de la participación de las mujeres dentro de los entes cooperativos en el período 1989-2012}

Sobre la posición de desventaja de la mujer en las cooperativas costarricenses, destacan dos estudios, uno realizado por APROMUJER y el otro por otra asociación, relacionada con el cooperativismo femenino, denominada la Asociación de mujeres en cooperación para el desarroIlo AMUCODE, dividido en dos Diagnósticos: el Diagnóstico sobre criterios que tienen las mujeres al tomar sus decisiones económicas y el Diagnóstico Género-Sensitivo sobre el comportamiento de los círculos de dirección en las cooperativas, aplicados contemporáneamente al mismo grupo de mujeres. En el primer estudio se ofrece una propuesta metodológica para abordar las causas de esa discriminación y se basa en el concepto asumido en la Convención sobre la Eliminación de toda forma de Discriminación contra la Mujer CEDAW, así Figueroa (s.a. p.20) hace referencia al término «discriminación» como toda distinción, exclusión o restricción basada en el sexo que tenga por objeto o resultado menoscabar o anular el goce o ejercicio de los derechos humanos y las libertades fundamentales de las mujeres, en las esferas política, económica, social, cultural y civil o en cualquier otra es- 
fera. Y agrega que la «discriminación» no es solo un tipo de conducta, sino que puede ser la omisión de la actuación, pese a la percepción de la existencia de limitaciones para la vida de las mujeres, en el caso concreto del Cooperativismo costarricenses, apunta Figueroa, M. (s.a. p. 10) que entre los elementos que se han definido como causas de discriminación de las mujeres se encuentran la legislación cooperativa y las prácticas cooperativistas.

Dada la poca información específica sobre la participación de las mujeres en el Cooperativismo costarricense, pues los censos ofrecen básicamente datos sobre número o porcentaje de participación de hombres y mujeres, en relación con todo el Cooperativismo o limitándola a los órganos directivos, se han tomado datos y opiniones de los estudios antes mencionados, como una forma de complementar la información necesaria para conocer, al menos parcialmente, por razones de los límites por fecha así como de la forma en que obtuvieron los resultados, que generalmente no ha sido de la totalidad de los miembros del Cooperativismo, o de la totalidad de las mujeres cooperativistas existentes en el momento de aplicar las encuestas.

\subsection{Limitada presencia en los cargos de dirección}

Figueroa lo disgrega en la falta de estímulo institucional para alentar la participación de las mujeres en roles de dirección, lo cual se explica en el estudio que las mujeres tienen menor nivel educativo y menor información para desempeñar cargos de dirección. Apunta Herrera (2009, p. 27) sobre la incidencia de las mujeres en las cooperativas, que el nivel educativo no es una garantía que subsane el grado de desconocimiento, pues la muestra del Diagnóstico aplicado por AMUCODE, refleja que a pesar del grado de educación superior de muchas mujeres tienen un conocimiento medio sobre finanzas. Concluye preliminarmente la autora, que el «desconocimiento de la economía no es sólo un problema de educación, también por la posición que la identidad de género tiene ante la economía como plantea Clara Coria».

A lo anterior se suman otras causas, relacionadas con los obstáculos de las mujeres para incidir en los círculos de dirección de las cooperativas, como son la falta de conocimiento y preparación sobre la dinámica empresarial y política de la cooperativa en la toma de decisiones; la falta de experiencia en el acceso, gestión y control de los recursos, además de las estructuras poco flexibles para la toma de decisiones en las cooperativas (Herrera 2009, pp. 32-33). Y la autora afirma que sucede esto porque las mujeres renuncian a la influencia en el uso y con- 
trol de recursos, bajo una apariencia sobre la inexistencia de relaciones no-conflictivas, pero que realmente son poco democráticas.

3.2. Los hombres si tienen capacidades innatas para dirigir a las mujeres

Herrera (2009, p. 35) hace referencia a otro Diagnóstico, denominado Género-sensitivo sobre el comportamiento de los círculos de dirección en las cooperativas, cuyo objetivo principal fue revelar cuáles eran las principales creencias, costumbres, actitudes constituidos en obstáculos para que las mujeres accedan, y adquieran influencia en los círculos de dirección de las organizaciones cooperativas. Los rasgos en orden de prioridad según sexo fueron los siguientes:

Cuadro dos

\section{Rasgos por sexo}

\begin{tabular}{ll}
\hline \multicolumn{1}{c}{ Mujeres } & \multicolumn{1}{c}{ Hombres } \\
\hline 1. Conocimiento & 1. Trayectoria \\
2. Trayectoria & 2. Conocimiento \\
3. Reputación e imagen & 3. Capacidad de mando \\
4. Relaciones personales & 4. Reputación e imagen \\
\hline
\end{tabular}

Fuente: Elaboración propia con base en datos del Diagnóstico de AMUCODE, noviembre de 2018.

Herrera (2009, p. 43) hace una análisis comparativo del cuadro, cuyo resultado es la consideración que para ambos sexos la trayectoria y el conocimiento ocupan un lugar importante entre los principales rasgos que se deben tener para incidir en las decisiones de la cooperativa. Además para las mujeres los rasgos de «reputación e imagen» y «relaciones personales» se asocian a la subjetividad e interacción de las personas; mientras en los hombres, los rasgos de "capacidad de mando» y de «reputación e imagen» se asocian, desde el punto de vista de la autora, a la detentación y el ejercicio del poder.

En las Consideraciones finales y recomendaciones se pueden extraer dos de ellas, de suma importancia pues mantienen vigencia al momento de elaborar este ensayo:

IV. Las mujeres cooperativistas se encuentran en una posición de desventaja respecto de los hombres, una vez que ingresan en los 
círculos de dirección, pues los hombres, en general, cuentan con más estudios, y la percepción les favorece en el grado de conocimiento sobre el funcionamiento de las cooperativas, además cuentan con mayor disponibilidad de tiempo para dedicar a la dirección por no tener las mismas responsabilidades extra laborales que las mujeres, e igualmente la percepción les adjudica mayor ejercicio del mando y de liderazgo, todo esto limita la incidencia efectiva de las mujeres.

$\checkmark$. Sucede además, que el análisis muestra un diferencial en el ejercicio del mando, pues tanto en la trayectoria para llegar a un puesto de dirección, como en el puesto mismo, los hombres tienden más a ejercer el mando, y esto es lo que favorece una sociedad con principios de dominación patriarcales, al mismo tiempo que las mujeres una vez en puesto de mando, destacan por su entrega y adaptación a las normas preestablecidas por tanto no juegan un papel activo para la inclusión de la perspectiva de género y las acciones por la equidad. (2009, p. 53)

Poco interés existe en el Cooperativismo costarricense por conocer las realidades anteriormente descritas y menos de corregirlas, mejorando la participación política de la mujer. En el 2014 el Comité regional de la mujer cooperativista de la ACI-AMERICAS solicitó información a los entes responsables y de representación de los diferentes Movimientos Cooperativistas del continente, sobre la participación de las mujeres dentro de las organizaciones cooperativas, para integrarlo a la Evaluación de estadísticas de Membrecía. Reporte de resultados de la Investigación Cuantitativa Organizaciones miembros de América Latina. (2014, p. 24) Los datos ofrecidos por el CONACOOP y disgregados en participación en órganos directivos así número de hombres y mujeres, fueron enviados en la siguiente forma:

Cuadro tres

Participación de hombres y mujeres en el cooperativismo costarricense

\begin{tabular}{lcc}
\hline \multicolumn{1}{c}{ Distribución } & Hombres & Mujeres \\
\hline Membresía en entes cooperativos & $55 \%$ & $45 \%$ \\
Puestos gerenciales & $100 \%$ & 0 \\
Mandos medios & $100 \%$ & 0 \\
Consejo de Administración & $38 \%$ & $63 \%$ \\
Comité de Vigilancia & Sin Información & \\
Comité de Educación & Sin Información & \\
Empleados & $55 \%$ & $45 \%$ \\
\hline
\end{tabular}

Fuente: Elaboración propia con base en datos del Conacoop 2018. 
En los datos antes transcritos hay omisiones graves en cuanto a la participación de la mujer en el cooperativismo nacional. Por ejemplo, sobre mujeres que ocupan el cargo gerencial y laboran en cooperativas de autogestión, según nombramientos inscritos en el Registro de Cooperativas del Ministerio de Trabajo antes de 2014:

- Cooperativa de Autogestión Industrial Panificadora San Carlos R.L. Coopepan, R.L.

- Cooperativa Autogestionaria de Servicios de Profesionales Multidisciplinarios R.L. Sula Batsu R.L.

- Cooperativa de Servicios a Mujeres Productoras y Microempresarias R.L (Coopemupro R.L.).

- Cooperativa de Servicios Múltiples de Pérez Zeledón R.L. Coopemuser. R.L.

- Cooperativa de Autogestión de Mujeres Innovadoras, R.L. (los anteriores datos fueron tomados de la página web Ministerio de Trabajo 2018).

- Cooperativa Molusqueros de Chomes R.L (Infocoop, 2015).

- Coopeande R.L, en el informe de liderezas de Cenecoop (2017).

También existía ya un estudio de Cárdenas y Mora (2012, pp. 58) basado en el Censo cooperativo de 2012 quienes apuntaron en relación la equidad de género, que:

El porcentaje de mujeres que participan en el quehacer cooperativo es muy amplio; el número de asociadas mujeres es solamente un $11 \%$ menor que el de los hombres. Asimismo, de los 4.928 cargos dirigenciales en los consejos de administración, comités de vigilancia y otros comités, 3.014 son varones y 1.824 son mujeres. En el cuadro 4 se aprecia la distribución por género en cada cuerpo directivo.

Cuadro 4

Distribución de cargos en función de género

\begin{tabular}{lrrrr}
\hline \multicolumn{1}{c}{ Cuerpo directivo } & Hombres & Mujeres & $\begin{array}{c}\% \text { de } \\
\text { mujeres }\end{array}$ & Total \\
\hline Consejo de Administración & 1.684 & 752 & 30,00 & 2.436 \\
Comité de Vigilancia & 742 & 423 & 36,00 & 1.165 \\
Comité de Educación y Bienestar Social & 535 & 577 & 52,00 & 1.112 \\
Otros comités & 143 & 72 & 33,00 & 215 \\
\hline Total de miembros & 3.104 & 1.824 & 37,75 & 4.928 \\
\hline
\end{tabular}

Fuente: Elaboración propia de los autores con datos de INFOCOOP de 2012. 
El Censo referido se presenta la distribución por sexo de las personas que ocupan el puesto de gerente de la cooperativa correspondían a 263 hombres (69\%) y 113 mujeres (30.1\%). (Cuadro 6, p. 25); en el cargo de Presidente del Consejo de Consejo de Administración de la cooperativa, habían 293 hombres (77.9\%) y 83 mujeres (22.1\%) (Cuadro 7, p. 25).

\subsection{Ubicación de la mayoría de las mujeres en la Educación Cooperativa}

En relación con el Diagnóstico sobre los criterios que tienen las mujeres al tomar sus decisiones económicas, Herrera (2009, pp. 7 y 13) responsable de AMUCODE y como parte de la ejecución del proyecto de fortalecimiento del liderazgo y de la organización de las mujeres para su incidencia en el movimiento cooperativo, explica que en relación con el nivel educativo de la población participante en el diagnóstico, cuyo umbral fue la educación secundaria —de 12 a 17 años de edad-, como un nivel mínimo de conocimientos necesarios para tener mayor entendimiento de la información necesaria relativa a la cooperativa e incidir en el nivel propositivo en la dirección de la misma, los datos fueron contundentes para mostrar la falta de estudios:

- La mayoría de las mujeres participantes no ha logrado concluir los estudios secundarios de la educación general básica, representaron el $52,38 \%$ de la muestra.

- El 30,95\% de las mujeres participantes contaba con educación superior, que fue un $11,9 \%$

— El 11,9\% obtuvieron el título de bachiller en educación secundaria.

Se indica en el Diagnóstico que la cooperativa debería ser un espacio de educación, en aplicación del quinto Principio cooperativo, para permitir que la dirigencia conozca a fondo la filosofía cooperativa, sus valores y principios así como dinámicas empresariales con sentido social (Herrera 2009, p. 14).

En el Censo cooperativo de 2012 dedicó espacio a recabar datos sobre capacitación, de tal modo que en el CUADRO II. Principales temas tratados en las actividades de capacitación y grupos a los que se dirigieron, la información obtenida se distribuyó en dos columnas, una relativa a los Principales temas tratados en los programas de la capacitación y la otra sobre la Cantidad de menciones, donde indica que se realizaron 6 cursos para capacitación en el 2011, de un total de 579. 
(2012, p. 18). Los esfuerzos de capacitación según Gráfico 10 p. 31 medidos porcentualmente, corresponden a $23 \%$ dado a los CUERPOS DIRECTIVOS; 22\% a la Gerencia; $22 \%$ al Personal administrativo; $21 \%$ a los asociados y $12 \%$ al Personal de producción. Un gran número contempló la Educación cooperativa (194 cursos); Administración y gerencia (84 cursos); Servicio al cliente y relaciones humanas (53 cursos). Sin que se encuentren cursos dedicados en forma específica a capacitación de las mujeres cooperativistas. En cuanto a la información recabada en el Censo sobre necesidades de capacitación, de los múltiples temas mencionados por las personas encuestadas, no existe ninguna mención a género, mientras se mantiene especial interés por obtener conocimiento en: los temas financieros y de contabilidad y Administración y planes estratégicos (2012, p. 31).

\section{Relación con los puestos ocupados por varones en instituciones públicas. Incidencia de las políticas públicas del país en relación con las oportunidades económicas y políticas ofrecidas a las mujeres}

La organización del Cooperativismo costarricense tiene en su cúpula la Asamblea de todas las cooperativas, la cual elige por cada uno de los tres sectores en que está dividido el Cooperativismo, diez representantes al Plenario y éste a su vez elige el Directorio que funciona en forma permanente. El Plenario también tiene la función de elegir o proponer ternas para elegir a los representantes del Cooperativismo, en diversos entes públicos relacionados con la producción agrícola o aspectos financieros que afectan al Cooperativismo, como es el caso de la elección de un representante a la Junta Directiva del Sistema nacional de riego y avenamiento SENARA, creado por Ley 6877 tiene entre sus objetivos lograr la función social de la propiedad agropecuaria y el agua, mediante la por la creación de distritos de riego y avenamiento, se efectué una modificación racional y democrática en la propiedad de la tierra, en organizaciones públicas o privadas y cooperativas que indudablemente corresponden al primer sector, denominado en la ley cooperativas agroindustriales.

Ante la Junta Directiva de la Caja Costarricense de Seguro Social, el CONACOOP, por la Ley n. 7983 (Ley de Protección al Trabajador, elabora una terna de representantes, y el Consejo de Gobierno elige a uno. En el caso del Programa Integral de Mercadeo Agropecuario PIMA, creado por Ley 20135, es el INFOCOOP quien propone la terna al Consejo de Gobierno. También el sector cooperativo tiene represen- 
tación en el Banco Popular y de Desarrollo Comunal, en su Asamblea de trabajadores y trabajadoras y posibilidad de nombrar representantes en su Junta Directiva Nacional, y otros órganos como son el Directorio, la Comisión de vigilancia, la Comisión de análisis financiero y balance social y sus Juntas de crédito — Ley No. 4351 de 11 de julio de 1969 y sus reformas-). Hasta el momento no existen datos estadísticos que reflejen la participación de alguna mujer cooperativista en las Juntas Directivas de esos entes.

La Junta Directiva Nacional es el máximo jerarca en su condición de órgano de dirección y cumple además las funciones de Asamblea de Accionistas de las Sociedades del Conglomerado. Desde la creación del Banco hasta 1990, sus miembros siempre fueron varones, hasta 1991 que la destacada cooperativista Marta Campos Méndez se incorporó como representante del Movimiento Cooperativo (APROMUJER, 2001, p. 119). La década transcurrió sin más participación femenina hasta la reforma de la ley de creación del Banco, por medio de la Ley 8322. Ley de Democratización de las Instancias del Decisión del Banco Popular y de Desarrollo Comunal (Reforma a la Ley Orgánica del Banco Popular, $\left.n .{ }^{\circ} 4351\right)$, donde gracias a la participación activa de otro de sus órganos, la Comisión de la mujer, se hizo obligatoria la participación femenina en sus órganos. La Asamblea tiene la obligación de elaborar la lista de directivos y directivas cumpliendo una cuota no menor de un $50 \%$ de mujeres.

El INFOCOOP, según su ley de creación, artículo 155, tiene como finalidad el fomento, promoción, financiamiento, divulgación y apoyo del cooperativismo en todos los niveles, propiciando las condiciones requeridas y los elementos indispensables a una mayor y efectiva participación de la población del país. El nombramiento en la Junta Directiva de esa institución se logra por medio de las elecciones en el Plenario del CONACOOP, donde se eligen a cuatro representantes, por períodos de dos años con posibilidad de reelección. De esa institución existen datos estadísticos sobre la participación de los varones y las mujeres cooperativistas en los 40 años de su existencia, contados a partir de 1973.

De esa institución existen datos estadísticos sobre la participación de los varones y las mujeres cooperativistas en los 40 años de su existencia, contados a partir de 1973.

En conclusión, las mujeres cooperativistas no han sido muy buenas negociadoras, una mujer no hace verano, es evidente que las mujeres cooperativistas hayan ha luchado por dejar otra compañera en el relevo, no ha abierto espacio para aumentar el número en la Junta Directiva del INFOCOOP. 


\section{Cuadro de integración de junta directiva del INFOCOOP, nombrados por el CONACOOP. Por género: hombres y mujeres}

\begin{tabular}{cccc}
\hline Año & Junta Directiva & Número varones & Número de mujeres \\
\hline 1973 a 1980 & I a XXIV & 4 & 0 \\
1982 & XXV y XXVI & 3 & 1 \\
1983 a 1998 & XXVII a XXXI & 4 & 0 \\
1999 & XXXII & 3 & 1 \\
2000 & XXXIII & 4 & 0 \\
2001 & XXXIV & 3 & 1 \\
2002 a 2010 & XXXV a XLVI & 4 & 0 \\
2011 & XLVII & 3 & 1 \\
2012 a 2013 & XLVIII a XLIX & 4 & 0 \\
\hline
\end{tabular}

Fuente: Cuadro elaborado por Ligia Roxana Sánchez Boza. Datos tomados de Infocoop 2018.

\section{Reforma a la ley de asociaciones cooperativas para introducir la equidad e igualdad de género}

El último intento de mejorar las condiciones antes expresadas, fue la presentación a la corriente legislativa del proyecto de ley denominado «Democratización de las diferentes instancias de decisión del movimiento cooperativo y creación del Comité nacional de las mujeres cooperativistas (CONAMUJER) al cual se le asignó el expediente n. ${ }^{\circ}$ 18.199, en el 2011, en el gobierno de la Presidenta de la República Laura Chinchilla. Nombre que fue variado en el siguiente período presidencial de Luis Guillermo Solís, conociéndolo como reforma de la Ley de asociaciones cooperativas y creación del Instituto nacional de fomento cooperativo, n. ${ }^{\circ} 4179$, del 22 de agosto de 1968 y adición de un nuevo título IV para creación de un Comité nacional para la equidad e igualdad de género y los Derechos Humanos de las mujeres del Movimiento Cooperativo. Este último obtuvo un Dictamen unánime afirmativo de la Comisión permanente especial de la mujer de la Asamblea Legislativa, el 25 de junio de 2013.

Los objetivos generales del proyecto mencionado han sido conseguir diferentes reformas a la actual Ley de asociaciones cooperativas, en primer lugar para incorporar a las mujeres en diferentes instancias del Cooperativismo y lograr el cumplimiento de los compromisos contraídos por el máximo ente decisorio del cooperativismo costarricense, que corresponden a las decisiones de los congresos nacionales que se realizan cada dos años —137 inciso e) de la LAC — donde se establece 
esa obligación de cumplir las disposiciones y resoluciones del Congreso Anual Cooperativo, asumidos democráticamente.

Es así que en la exposición de motivos del proyecto mencionado, el ente promotor de estas reformas denominado Comité Nacional de la Mujer Cooperativista (CONAMUJER) logró la aprobación en el XII Congreso Nacional Cooperativo la aprobación de una política de igualdad y equidad de género en el movimiento cooperativo, avalada totalmente por los delegados del Congreso junto con un mandato de su ejecución. ${ }^{1}$

En forma similar al avance logrado en el Banco popular y de desarrollo comunal, en 2000, indica Sánchez (2002, s. p.) este grupo de liderezas cooperativistas han buscado que el movimiento cooperativo democratice órganos decisorios en forma paritaria y que en sus decisiones se incorpore la perspectiva de género, y lograr la paridad en todos los órganos de decisión del más alto nivel del movimiento cooperativo y la obligatoriedad de cumplir la política de igualdad y equidad de género del sector.

En segundo lugar, otro objetivo es lograr el fortalecimiento del Comité Nacional de las Mujeres Cooperativas (CONAMUJER) en el tanto que es la organización de máxima representación política de las mujeres cooperativistas; cuya creación data desde 1987 por mandato de los IV y V Congresos Nacionales Cooperativos.

Entre las reformas más importantes se encuentran:

- La obligación de nombrar mujeres en la Junta Directiva del INFOCOOP.

- Obligación de las uniones, federaciones y las confederaciones de cooperativas de respetar la igualdad y equidad de género, en su constitución, administración y funcionamiento.

- La creación de CONAMUJER como un ente público no estatal, con personería jurídica y plena autonomía de administración y organización financiera, de máxima representación política para la igualdad y equidad de género del movimiento cooperativo de Costa Rica.

- Incorporación a la Junta Directiva del INFOCOP de una representante de CONAMUJER, que impulsará e incidirá en la incorporación de la perspectiva de género.

El proyecto en estudio ha contado con el apoyo de la Comisión legislativa de la mujer, pero ha enfrentado una fuerte resistencia por 
parte de los directivos del Consejo Nacional de Cooperativas, que llegaron al punto de interponer un recurso de amparo ante la Sala Constitucional porque consideraron que algunos artículos violaban la Constitución Política.

Los actores sociales del cooperativismo que han intervenido ante la Comisión mencionada han sido:

- El Consejo Nacional de Cooperativas se opone rotundamente a la creación de otro órgano especializado en asuntos de la mujer con independencia financiera y reformas a la Ley de asociaciones cooperativas para incluir el principio de equidad e igualdad de género en todas las instancias cooperativas. El CONACOOP manifestó que no es necesaria a la Ley de Cooperativas que incluya textualmente el principio de igualdad en el movimiento cooperativo debido a que el art. 3, inciso e) implícitamente lo contiene al plantear la no discriminación de ninguna índole.

- La Asociación de mujeres en cooperación para el desarroIlo (CONAMUJER), es la única organización de mujeres que defiende el proyecto, en el tanto que el Comité de la mujer cooperativista, según su percepción ha sido neutralizado como reacción del CONACOOP ante la presentación del proyecto de Ley:

La cúpula del Movimiento Cooperativo, destituyó el Comité ante la presentación del proyecto de ley (Expediente 15810) y fue desintegrada la Junta Directiva del Comité Nacional.

Cabe anotar que la acción de Inconstitucionalidad contra varios de los artículos de la ley en 18199, argumentando que en órganos privados no se debe obligar a tener la paridad, pero la Sala Constitucional la declaro sin lugar, por Voto N. 4630-2014, pues consideró que la normativa impugnada no es inconstitucional, más bien, consideré que constituye una medida de acción afirmativa, que se sustenta en la fuerte promoción de una mayor participación de la mujer en todos los campos de la vida social política y económica, establecida principalmente, por el Derecho Internacional de los Derechos Humanos y que, como tal, no resulta violatoria del principio a la libertad, el derecho a la igualdad, la libertad de asociación, la libertad sindical o los principios de razonabilidad y proporcionalidad.

En Costa Rica, considera la autora, las cooperativas nacen con base en las disposiciones de la Ley de asociaciones cooperativas, como entes de derecho privado con autonomía funcional y bajo los Principios establecidos en el artículo 3, el cual amplía su contenido, por medio 
de las normas de integración normativa establecidas en el artículo 131 de la misma ley. Ese artículo indica que los asuntos cooperativos, también, deben ser analizados con base en los Principios generales del Derecho Cooperativo, los cuales han sido establecidos en los Congresos del máximo organismo mundial del Cooperativismo como es la Alianza Cooperativa Internacional. Entre los Principios generales del Derecho Cooperativo se encuentra el reconocimiento del principio de Participación democrática en todas las instancias del Cooperativismo nacional, por medio de la equidad de género, lo cual lleva a que cada Movimiento cooperativo nacional tome las decisiones políticas para lograr el cumplimiento de tales principios.

Los mandatos de los Congresos Cooperativos tienen rango de ley porque así lo reconoce el artículo 137 inciso e) mencionado. Una vez aprobados válidamente corresponde al Consejo nacional de cooperativas cumplir con las decisiones acordadas, en forma democrática, por todos los representantes de las cooperativas constituidos en el Congreso Cooperativo, por esa razón, las modificaciones a la normativa cooperativa nacional propuestas en el proyecto de Ley N. ${ }^{\circ} 18.199$, responden en forma fiel a las órdenes dadas por la Asamblea de delegados cooperativistas, a través de los Congresos cooperativos, por lo cual los diputados y diputadas están legitimados dentro del bloque de legalidad cooperativa, a dictar la normativa legal necesaria para su cumplimiento.

El último proyecto referido, fue enviado a archivo por aplicación del plazo cuatrienal, por parte de la Presidenta de la Asamblea Legislativa, el 11 de octubre de 2018. Al presente un grupo de diputadas acogieron nuevamente el proyecto de ley y han mantenido el mismo contenido del anterior.

\section{Conclusiones}

Existen los conceptos de igualdad, equidad de género, discriminación por razón de género en diferentes instrumentos legales así como en acuerdos de diferentes instancias tanto públicas como privadas. Por ejemplo el PIEG en el ámbito nacional o los compromisos de los Comités de la mujer cooperativista que se han creado en el seno de la $\mathrm{ACl}$ América, como fue la aprobación de la Plataforma de acción de mujeres cooperativistas del Continente Americano, en 1997, en cuyos enunciados se han reforzado la aspiración de la $\mathrm{ACl}$ de contribuir al cambio en las relaciones desiguales e injustas entre los géneros al interno de las cooperativas. En la CCC-CA y Sudamérica, o bien, decisio- 
nes incorporadas como acuerdos en los Congresos Cooperativos, en el ámbito del cooperativismo nacional. Sin embargo, la infrarrepresentación política de las mujeres se mantiene por décadas.

Los Censos Cooperativos así como los Diagnósticos elaborados ofrecen el panorama negativo en el avance de la obtención de derechos en el campo político de las mujeres cooperativistas. Tampoco ha ayudado el reconocimiento que les da el Segundo principio cooperativo, la redacción inclusiva de las Notas de interpretación para los Principios cooperativos, emitidas por la $\mathrm{ACl}$, en el 2012, dado que hay obstáculos que están en el fondo de la posición de la mujer en la sociedad, tales como problemas de capacitación para ejercer el poder político, ausencia de políticas inclusivas por parte de los entes cooperativos de representación y defensa, empezando por el CONACOOP, pero que también se debe incluir a entes de segundo y ulterior grado como son las uniones y federaciones cooperativas.

Ha sido manifiesto el poco interés por no decir ausente, del Cooperativismo costarricense en cuanto a la apertura de espacios de participación política de la mujer, se hizo manifiesto en 1991 cuando se aplicó por parte de APROMUJER, y no hubo mucha colaboración con la aplicación de las encuestas. En igual forma, en este siglo los datos ofrecidos por el CONACOOP al Comité regional de la mujer cooperativista de la ACI-América, fueron presentados sin hacer un estudio de los registros del Ministerio de Trabajo en su Dirección de Cooperativas, como una fuente de información primaria de gran relevancia para dar informes sobre la posición de la mujer cooperativista. También AMUCODE hizo evidente, con sus diagnósticos, que es esencial la capacitación para impulsar la participación política de la mujer, sin embargo, el mismo Censo cooperativo de 2012 demostró que no hay interés en la realización de capacitaciones con ese objetivo, pues de la información recabada en el Censo sobre necesidades de capacitación, de los múltiples temas mencionados por las personas encuestadas, no existe ninguna mención a género, mientras se mantiene especial interés por obtener conocimiento en: los temas financieros y de contabilidad y Administración y planes estratégicos.

En las representaciones del Cooperativismo en entes públicos no hay referencias para conocer si una mujer ha obtenido tal representación, salvo en el INFOCOOP, que como presenta el cuadro incluido, sólo ha habido en pocos años, una sola mujer nombrada por el CONACOOP como miembro de la Junta Directiva del Instituto.

Las últimas decisiones que han tomado algunas mujeres cooperativistas, por la falta de eco de sus reclamos de más apertura para su participación política en el Cooperativismo, ha sido presentar proyectos de 
reforma a la LAC, los cuales no han tenido fortuna, y casi cumplen una década de discusiones en el campo legislativo, y se han dado posiciones en el CONACOOP que llegó al punto de desintegrar la Junta Directiva del Comité Nacional de la Mujer cooperativista para obstaculizar su lucha por lograr la reforma legal mencionada. Tampoco se ha dado el reconocimiento a la fuerza de los mandatos de los Congresos Cooperativos dirigidos a mejorar la condición de la mujer, en relación con su participación política y que tienen rango de ley.

En este momento, el último proyecto de reforma a la LAC fue enviado a archivo por parte de la Presidenta de la Asamblea Legislativa, el 11 de octubre de 2018. Lo cual provocó que otro grupo de mujeres gestionara ante las nuevas diputadas -el gobierno cambio en mayo 2018 - para incorporarlo de nuevo a la corriente legislativa. En síntesis la lucha de la mujeres cooperativistas de Costa Rica ha sido extensa y profunda, pero le ha faltado apoyo político dentro y fuera del Movimiento Cooperativo.

\section{Bibliografía}

Alianza Cooperativa Internacional para las Américas (2009). Ley Marco para las cooperativas de América Latina. Ed. Alianza Cooperativa Internacional para las Américas. San José, Costa Rica.

Alianza CoOperativa Internacional (2012). Notas de interpretación para los Principios cooperativos. Recuperado el 11-11-2018 de https://www. aciamericas.coop/IMG/pdf/guidance_notes_es.pdf.

Asamblea Legislativa (1984). Convección para la Eliminación de toda Forma de Discriminación contra las Mujeres (CEDAW). Costa Rica.

Asamblea Legislativa 1983). Ley de Creación del Servicio Nacional de Aguas Subterráneas, Riego y Avenamiento (SENARA) No. 6877. (Recuperado de: http://www.senara.or.cr/acerca_del_senara/marco_legal/leyes_ senara/6877\%20Ley\%20de\%20creacion\%20del\%20SENARA.pdf

AsAMBLEA LEGISLATIVA (1983). Ley orgánica del Instituto nacional de aprendizaje. N. 6868. Recuperado el 11 de noviembre de 2018: http://www.pgrweb. go.cr/scij/Busqueda/Normativa/Normas/nrm_texto_completo.aspx?param $1=$ NRTC \&nValor1=1 \&nValor2=36651\&nValor3=38641\&strTipM=TC

Asociación Programa nacional de Asesoría y Capacitación para la Mujer, APROMUJER. (1991). Diagnóstico, participación de la mujer en el movimiento cooperativo costarricense, San José, Costa Rica.

Asociación Programa nacional de Asesoría y Capacitación para la Mujer, APROMUJER, (2001) Escritos de M. artículos, discurso y pensamientos Marta Campos Méndez. S. Ed. Heredia, Costa Rica.

Asamblea Legislativa (1943) Ley constitutiva de la Caja Costarricense de Seguro Social n. ${ }^{\circ}$ 17. Ed. Imprenta Nacional. San José, Costa Rica. 
Asamblea Legislativa (1968). Ley 4179 Ley de asociaciones cooperativas y creación del Instituto Nacional de Fomento Cooperativo. Recuperado el 3 de marzo de 2018 de http://www.infocoop.go.cr/cooperativismo/legislacion/ ley_4179_conexas.pdf

Asamblea legislativa (1956). Ley Orgánica del Consejo Nacional de Producción N. 2035. San José, Costa Rica.

Asamblea Legislativa (1983). Ley orgánica del Instituto nacional de aprendizaje. N. 6868. Recuperado de: http://www.pgrweb.go.cr/scij/Busqueda/Normativa/Normas/nrm_texto_completo.aspx?param1=NRTC\&nValor1=1\&nValor 2=36651\&nValor3=38641\&strTipM=TC

AsAmbleA LeGISLATIVA (2002). Ley 8322. Ley de Democratización de las Instancias del Decisión del Banco Popular y de Desarrollo Comunal (Reforma a la Ley Orgánica del Banco Popular, n. ${ }^{\circ}$ 4351). Recuperado de: 2018.http://www. pgrweb.go.cr/scij/Busqueda/Normativa/Normas/nrm_texto_completo.aspx? param 1 =NRTC \&nValor1=1\&nValor2=49559\&nValor3=53016\&strTipM=TC

CÁrdenAs, G. y Mora, A. (2012) Visión panorámica del sector cooperativo en Costa Rica. Una larga historia del sector. Ed. Alianza cooperativa internacional y Organización Internacional del Trabajo. La Paz, Bolivia. Recuperado el 12 de octubre de 2017 https://www.aciamericas.coop/IMG/pdf/ wcms_185287.pdf

CENECOOP R.L. (2017) Gerentes líderes de INCAE. Recuperado el 11 de noviembre de 2018 de https://www.youtube.com/watch?v=uTFepNF6pKU.

Comité Regional Americano de Mujeres Cooperativistas ACI Américas (1996). Plataforma de acción de mujeres cooperativistas del continente americano. San José, Costa Rica. Recuperado el 15 octubre de 2018 de http://www. aciamericas.coop/IMG/cramc1996.pdf

Confederación de Cooperativas de Centroamérica, El Caribe y Sudamérica (2010) Programa Regional de Equidad Género y Generacional (PREG) recuperado el 11 de noviembre de 2018 de http://www.ccc-ca.com/programasproyectos.php

Herrera, J. (2009). Algunos elementos a tomar en cuenta para que las mujeres logren incidencia en los espacios de dirección de las cooperativas. Asociación de mujeres en cooperación para el desarrollo (AMUCODE) y Centro cooperativo sueco. Oficina regional para América Latina. Ed. Royo. Costa Rica.

Instituto Nacional de Fomento Cooperativo (2015). Cooperativa Molusqueros de Chomes R.L en Surgen seis cooperativas en territorios costeros de la península de Nicoya. Recuperado el 11 de noviembre de 2018 de http://www. infocoop.go.cr/enterese/noticias/2015/febrero/febrero_4.html

Instituto Nacional de las Mujeres (2007). Política Nacional para la lgualdad y Equidad de Género 2007-2017. Instituto Nacional de las Mujeres. San José.

Ministerio de Trabajo y Seguridad Social (2018). Expediente registrado en el Departamento de Organizaciones Sociales de las cooperativas.

- Cooperativa de Autogestion industrial Panificadora San CarLOS R.L. COOPEPAN, R.L. Recuperado el 11 de noviembre de 2018 
de http://201.192.54.110/weblink/DocView.aspx?dbid=0\&id= 495\&page $=77 \& \mathrm{cr}=1$

- Cooperativa Autogestionaria de Servicios de Profesionales Multidisciplinarios R.L. SULA BATSU R.L. Recuperado 11 de noviembre de 2018 de http://archivodigitalos.mtss.go.cr/WebLink/DocView. aspx?dbid=0\&id=6\&page $=130 \& \mathrm{cr}=1$

- Cooperativa de Servicios a Mujeres Productoras y Microempresarias R.L (COOPEMUPRO R.L.). Recuperado 11 de noviembre de 2018 de http://201.192.54.110/WebLink/DocView. aspx?dbid=0\&id=400\&page=135 $\& \mathrm{cr}=1$

- Cooperativa de Servicios Multiples de Pérez Zeledon R.L. COOPEMUSER. R.L. Recuperado 11 de noviembre de 2018 de http://archivodigitalos.mtss. go.cr/WebLink/DocViewM.aspx?id=279\&dbid=0

Programa Estado de la Nación IV Censo Nacional Cooperativo - $1 .{ }^{a}$ ed.- San José, C.R. Instituto Nacional de Fomento Cooperativo 2012. Recuperado 14 de noviembre de 2018 de https://www.aciamericas.coop/IMG/pdf/ censo_cooperativo_cr2012.pdf

Sala constitucional (2014) Voto N. 4630-2014 de las 16 hrs del 2-4, San José, recuperado de http://jurisprudencia.poder-judicial.go.cr/SCIJ_PJ/busqueda/ jurisprudencia/jur_Documento.aspx?param 1=Ficha_Sentencia\&nValor $1=1$ $\& \mathrm{cmbDespacho}=0007 \&$ txtAnno $=2014 \&$ strNomDespacho $=$ Sala\%20Consti tucional\&nValor $2=616062 \& \mid$ Resultado $=1 \& \mid$ VolverIndice $=$ IndiceDespSent $\&$ param01=Sentencias \%20por\%20Despacho\&param2=42\&strTipM=T\&strD irSel=directo

SÁnCHEZ, L.R. (2002). "Antecedentes de la legislación cooperativa costarricense». Boletín de la Asociación Internacional de Derecho Cooperativo. ISSN: 1134-993X, Núm. 43/2009, Bilbao, págs. 73-91 91. Recuperado el 3 de marzo de 2018 de file:///C:/Users/Roxana/Downloads/Dialnet-Anteceden tesDeLaLegislacionCooperativaCostarricens-3074484.pdf

SÁnCHEZ, L.R. (2005). ¿Cómo se forjó la primera ley de Costa Rica donde se establece la paridad en las instancias politicas del Banco Popular y de Desarrollo Comunal? Recuperado de: neticoop.org.uy 